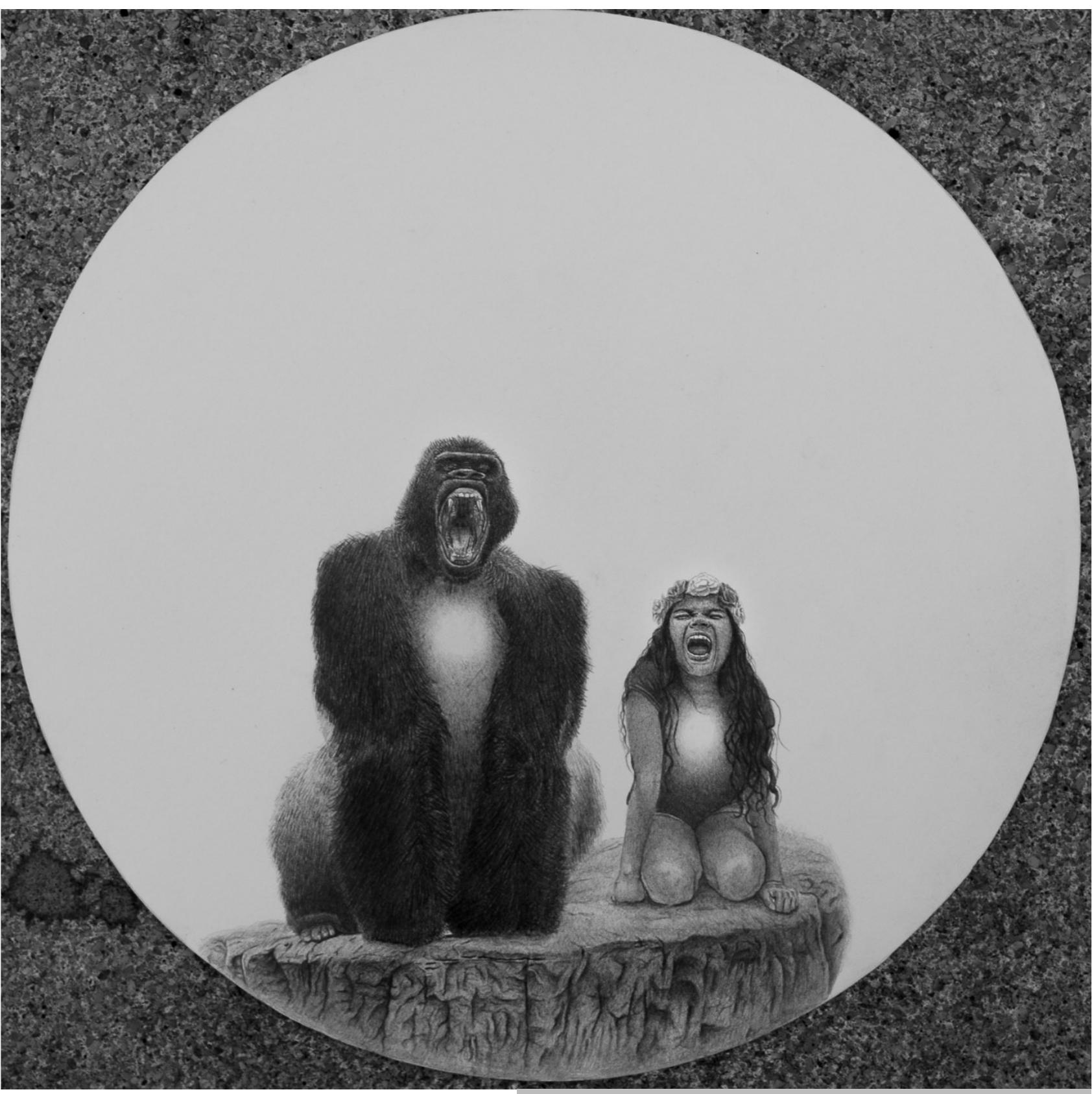

Sin título

De la serie Un viaje hacía el origen

Grafito sobre papel

$12 \times 12 \mathrm{~cm}$

2019

Medellín 


\title{
Álvaro García Linera y las contradicciones entre Estado y democracia*
}

\author{
Sergio Felipe Ayala Ruiz (Colombia)** \\ Silvia Juliana Quintero Erasso (Colombia)***
}

\section{Resumen}

Este artículo explora la forma en que Álvaro García Linera responde a la tensión entre política desde el Estado y política más allá del Estado emergida en el proceso político boliviano durante lo que va corrido del siglo xxı. Una vez explicados los términos de esta tensión, este texto sostiene que el distintivo en la propuesta de García Linera radica en una comprensión sobre la relación entre democracia y Estado caracterizada por actualizar, en el presente latinoamericano, la idea de que una acción política transformadora puede estar situada no solamente en uno de estos dos ámbitos, sino en los dos. En la propuesta teórica de García Linera emergen, sin embargo, distintas ambigüedades que permiten sugerir, al final, grandes dificultades para pensar una política transformadora más allá del Estado que no esté subordinada a las posibilidades de transformación desde lo Estatal.

\section{Palabras clave}

Teoría Política; Estado; Democracia; Socialismo; García Linera, Álvaro; América Latina.

Fecha de recepción: febrero de 2019 - Fecha de aprobación: junio de 2019

\section{Cómo citar este artículo}

Ayala Ruiz, Sergio Felipe y Quintero Erasso, Silvia Juliana. (2019). Álvaro García Linera y las contradicciones entre Estado y democracia. Estudios Políticos (Universidad de Antioquia), 56, pp. 199-220. DOI: 10.17533/udea.espo.n56a09

\footnotetext{
* Este artículo se realizó en el marco del proyecto de investigación colectiva Movimientos sociales y subjetividades: desafios teóricos desde América Latina, del grupo de investigación Teoría Política Contemporánea (Teopoco), Universidad Nacional de Colombia.

${ }^{* *}$ Politólogo. Historiador. Magíster en Filosofía. Magíster en Economía Política. Miembro del grupo de investigación Teoría Política Contemporánea (Teopoco), Universidad Nacional de Colombia. Correo electrónico: sfayalar@unal.edu.co

*** Politóloga. Miembro del grupo de investigación Teoría Política Contemporánea (Teopoco), Universidad Nacional de Colombia. Correo electrónico: sjquinteroe@unal.edu.co
} 


\title{
Álvaro García Linera and the Contradictions Between State and Democracy
}

\begin{abstract}
This article explores Álvaro García Linera's conceptualization on the tension between politics from the State and politics beyond the State, tension emerged in the $21^{\text {st }}$ Century Bolivian politics. This study explains, first, the terms of the tension, and second, it argues that the distinctive character of García Linera's proposal lies in a particular understanding of the relationship between democracy and the State. This relationship is characterized by updating, in the Latin American present, the idea that any transformative political action should be unfolded not only from one of these two spheres, but actually from both. However, different ambiguities emerge in García Linera's theoretical proposal that prevents thinking of a transformative politics beyond the State that is not subordinated to the possibilities of transformation from the State.
\end{abstract}

\section{Keywords} America.

Political Theory; State; Democracy; Socialism; García Linera, Álvaro; Latin 


\section{Introducción}

El siglo xxı ha transcurrido para Bolivia en medio de un agitado proceso de reorganizaciones políticas e institucionales. De 2000 a 2005 tuvieron lugar distintos procesos de movilización popular en defensa de la hoja de coca, así como la llamada guerra del agua de Cochabamba en 2000 y la guerra del gas en 2003, concentrada especialmente en la ciudad de El Alto y cuyo resultado más conocido es el de la expulsión del presidente Gonzalo Sánchez de Lozada del Palacio de Gobierno. Luego, la consolidación de una fuerza electoral alternativa representada en el Movimiento al Socialismo (MAS), cuya voluntad de poder estatal se hizo real en la elección de Evo Morales en 2006 (Prada, 2009, p. 141). Desde entonces, la emergencia de una inusitada actividad de movilización social y de un gobierno de izquierda que ha intentado desarrollar su mandato navegando en un mar de profundas contradicciones culturales, geopolíticas y socioeconómicas, entre apuestas por alcanzar reformas significativas y la frecuente imposibilidad de romper con lo heredado (Farthing y Kohl, 2014; Webber, 2011; Crabtree, 2008; Prada, 2009).

Sin lugar a duda, el proceso político boliviano no se agota en el gobierno del MAS y ciertamente resultaría reduccionista afirmar lo contrario. Más bien, la elección de Morales «es en parte el resultado de los grandes desajustes en el sistema político boliviano que entre 2000 y 2005 prácticamente borraron del tablero a los partidos y liderazgos tradicionales» (Cunha Filh, 2014, p. 138). No obstante, sería igualmente estrecho reducir todo el proceso político en Bolivia única y planamente a la «movilización social de base», pues se estaría desconociendo la compleja experiencia institucional acaecida en Bolivia en las dos últimas décadas. Lo cierto es que el proceso político-institucional ocurrido durante el gobierno de Morales, «por lo bueno o por lo malo, será seguramente y por mucho tiempo referencia para cualquiera que busque analizar y comprender la política de Bolivia» (Cunha Filh, 2014, p. 137). De cara a ello, la tensión que aparece entre el Estado y los movimientos sociales bolivianos constituye uno de los problemas más notables a los que ha tenido que enfrentarse la teoría política en Latinoamérica.

En este marco que se ubica una de las respuestas teóricas más visibles y relevantes a tal tensión, la referida a buena parte de la producción intelectual 
de Álvaro García Linera, ${ }^{1}$ vicepresidente de Bolivia desde 2006. Como intelectual en ejercicio del poder estatal, el pensamiento de García Linera está marcado por una permanente reflexión sobre el Estado en perspectiva de su relación con los otros ámbitos de lo social. Más específicamente, y en particular después de su llegada a la Vicepresidencia boliviana, García Linera se ha enfrentado y ha elaborado una respuesta desde la teoría política a una serie de contradicciones surgidas el seno de lo que él llama un Estado revolucionario en el país andino: Estado y movimientos sociales; democratización y centralización de las decisiones; nación-bloque popular y pluralidad indígena; intereses generales e intereses particulares; entre otras.

Un punto privilegiado a partir del cual él ha abordado este repertorio de contradicciones aparece en la pregunta por la relación entre democracia y Estado. Especialmente durante y después del proceso de instauración de la Nueva Constitución Política del Estado Plurinacional de Bolivia (2009), García Linera se ha interesado por pensar las implicaciones — puestas en términos de contradicciones- de encarar la crisis del viejo Estado dominante, asumir un proceso transicional de estatalidad y, eventualmente, construir un Estado nuevo (García Linera, 2015b). De allí, por ejemplo, la importancia y amplia acogida de obras como Las tensiones creativas de la revolución (García Linera, 2011), en donde el autor pretende explorar e identificar una serie de «tensiones» políticas, culturales y económico-sociales que confrontan el ejercicio del gobierno del Movimiento al Socialismo (MAS) desde 2006. En este texto, el autor señala que, para el caso de la «quinta fase del proceso revolucionario» — después de la «toma del poder» por parte del bloque popular emergente-, la «revolución democrática cultural» debe enfrentar una serie de contradicciones principales y secundarias (García Linera, 2011, p. 12). Contrario a ser marginal o accesorio, el pensamiento sobre las contradicciones resulta prioritario en la agenda teórica y política de García Linera como parte del proceso revolucionario mismo.

Así, lejos de entregarse a una reflexión autorreferente sobre el aparato estatal o la legitimación de una razón de Estado, su pensamiento sobre este

\footnotetext{
${ }_{1}^{1}$ Álvaro García Linera es un político y pensador boliviano, autor de varios textos frecuentemente citados en la teoría política latinoamericana contemporánea, tales como La potencia plebeya (2008), Las tensiones creativas de la revolución (2011) o Forma valor-forma comunidad (2009). Formó parte de la fundación del Ejército Guerrillero Tupaj Katari en la década de 1980 y del Grupo Comuna en Bolivia. Por la relevancia teórica y política tanto en Bolivia como en el continente, en este artículo se ha optado por estudiar la obra reciente del autor.
} 
último está directamente conectado con la pregunta por cómo entran a jugar las determinaciones sociales, culturales, de clase, económicas y políticas en y hacia el ámbito de lo estatal. Todas estas determinaciones que, en principio, parecen un afuera del Estado, están de hecho vinculadas con una idea singular de democracia en la obra del autor. Sin ir demasiado lejos, esta es la interpretación que ofrece García Linera frente al hecho de que la sociedad boliviana en las últimas décadas se ha pensado y construido «democráticamente» en un permanente desbordar toda idea de democracia entendida exclusivamente como una forma de gobierno estatal o de «técnica institucional», tanto en las experiencias organizativas, comunitarias y de movilización social como en el trabajo de distintos intelectuales bolivianos contemporáneos.

De cara a todo lo anterior, en este texto se exploran los contornos más generales de la conceptualización hecha por García Linera sobre la relación entre Estado y democracia para responder a la compleja red de tensiones surgidas entre el proceso político boliviano durante el gobierno del MAS. En este marco, el texto presenta dos objetivos principales: por un lado, reconstruir de manera más o menos sistemática los principales elementos conceptuales del planteamiento de García Linera; por el otro, examinar algunas líneas críticas sobre la forma en que estos planteamientos se relacionan y dialogan, en tensión, con los problemas concretos en el seno de la sociedad boliviana a los cuales busca responder. Para ello es crucial incorporar también las voces teórica y políticamente disidentes del oficialismo del MAS (Rivera Cusicanqui, 2012; Modonesi, 2012; Tapia, 2010; Prada, 2009; 2013, enero 16; Webber, 2015).

Las siguientes páginas se concentran en una selección de textos producidos durante y después de la instauración del nuevo texto constitucional en Bolivia. Para ello, se considera que las claves interpretativas apostadas por García Linera, a propósito del Estado en transición y el Estado nuevo en clave democrática, emergen de manera más decisiva en tal coyuntura para fortalecer el sustento teórico del proceso político dado a partir de la apuesta constitucional del gobierno de Evo Morales.

La tesis de este artículo es que, si bien Álvaro García Linera ofrece una comprensión más compleja sobre lo que implica una acción política transformadora desde la estatalidad en la Bolivia del siglo Xxı, hay una serie de ambigüedades no resueltas que minan significativamente el potencial 
de pensar formas de democracia, comunidad y acción política más allá del Estado o no exclusivamente desde el Estado. Esto pese a que en la misma obra del autor hay un insistente reconocimiento por plantear que la estatalidad es solo un momento de la democracia y que, en la perspectiva de un «socialismo democrático», es la democracia la que permanentemente revoca, configura y reconfigura lo estatal. Allí, por demás, es donde desempeña un rol crucial el significativo brazo crítico —organizaciones y teóricos no oficialistas-que se ocupa de esta «no suficientemente explorada» dimensión de lo democrático en Bolivia más allá del Estado.

En este orden de ideas, este texto reconstruye, en primer lugar, algunas cuestiones nucleares sobre la conceptualización de Estado que ha venido consolidando García Linera en los últimos años, en especial, puntualizando en su concepción sobre el Estado como relación social; en segundo lugar, se reconstruyen algunas consideraciones generales hechas por el autor respecto a lo que significa para él la democracia, en especial, en cuanto a la dimensión práctica y contingente de esta última y la necesidad de pensar «nuevas democracias»; finalmente, se retoma la forma en que el autor ha venido trabajando lo que a su juicio debe ser una «vía democrática al socialismo», para luego plantear una serie de conclusiones críticas.

Resulta relevante mencionar que este artículo se ocupa principalmente de las respuestas teóricas de García Linera a los retos arriba expuestos. No se trata de contrastar sus planteamientos teóricos con los desarrollos históricos más concretos de su práctica como dirigente político en Bolivia, si bien se reconoce que esta tarea debe ser objeto de un texto posterior. ${ }^{2}$

\section{Estado}

Antes de entrar en materia en esta primera sección cabe decir que, por cuestiones de espacio, este artículo no puede ocuparse de dos temarios importantes a propósito de la comprensión del autor sobre el Estado, los cuales tienen que ver con dos referentes teóricos de central importancia en

\footnotetext{
${ }^{2}$ García Linera es probablemente el primer intelectual marxista en llegar al poder en el siglo XXI, frente a lo cual «es necesario considerarle tanto en su producción teórica como en su hacer político, además del contexto boliviano de cual es inseparable» (Cavooris, 2015, p. 5). Sin embargo, en este artículo se resalta cómo específicamente desde la teoría el autor ha intentado responder a una serie de interrogantes en la sociedad boliviana. Esto implica más un ejercicio de énfasis en el análisis y en ningún sentido una apuesta por compartimentar la integralidad de la experiencia política del autor, mucho menos se espera profundizar en las implicaciones de separar teoría y práctica.
} 
sus interpretaciones: por un lado, la influencia de Gramsci, en particular, a través de la construcción de hegemonía y de formas de disputa del sentido común desde el Estado, apelando a liderazgos simbólicos con repertorios culturales que subviertan la tradición (García Linera, 2015b); por otro lado, con respecto a Vladimir Lenin, la reflexión de cómo pensar un tiempo transicional del Estado en donde no basta con apelar a la construcción de hegemonía, sino también a formas de intervención más explícitas en contra de las élites tradicionales en ejercicio del poder, en especial en función de lograr recrear lo que el autor Ilama un punto de bifurcación hacia un nuevo gobierno (García Linera, 2013; 2015a).

Dicho esto, una interpretación interesante sobre cómo se ha dado en concreto la tensión entre política de Estado y política más allá del Estado en los procesos de transformación en Bolivia ha sido la de Raquel Gutiérrez Aguilar (2017), quien señala que en el país Andino, antes y durante la llegada del gobierno de Evo Morales, pueden distinguirse dos horizontes políticos «confrontados y en competencia, con posibilidades intermitentes pero siempre plagadas de tensión y de colaboración entre sí» (p. 37). Por un lado, una perspectiva nacional popular más centrada en la construcción de Estado y en cambiar la relación entre sociedad y gobierno; por otro lado, una perspectiva política comunitaria-popular, cuya principal característica fue la de una apuesta por recrear una «reapropiación colectiva de la riqueza material disponible» (p. 38), incluyendo la construcción de mecanismos de decisión y gestión de tal riqueza. Si bien esta última no corresponde a una postura antiestatista, para la autora este segundo horizonte no es de ningún modo «la reconstrucción de ningún tipo de Estado» (p. 38).

Aunque desde una orilla distinta a la de Gutiérrez, García Linera también ha buscado generar sus propias respuestas frente a esta tensión, poniendo el énfasis en producir una lectura teórica singular y novedosa del Estado, partiendo de una pregunta central: ¿por qué el Estado para la revolución y cómo hacer política revolucionaria desde el Estado en Bolivia?³ Uno de los propósitos centrales del autor en su comprensión sobre el Estado

\footnotetext{
${ }^{3}$ Vale la pena resaltar que la asunción de un Estado revolucionario es mención propia del autor en varios de sus textos. Por ejemplo, en Las tensiones creativas de la revolución, el autor analiza los cursos de la democracia boliviana posrevolucionaria, frente a lo cual afirma que «el pueblo boliviano ha consolidado su unidad histórica en torno a un único proyecto de Estado, economía y sociedad» (García Linera, 2011, p. 07). El carácter revolucionario o no del proyecto de gobierno del MAS tiene, en todo caso, distintos matices en las izquierdas y la producción teórica bolivianas y latinoamericanas.
} 
tiene que ver con rechazar los planteamientos que separan la existencia histórica del Estado de la de los otros ámbitos de lo social. Al respecto, una discusión célebre es la entablada con John Holloway (2002) y su tesis de "cambiar el mundo sin tomar el poder». Para García Linera, propuestas como la de Holloway conciben al Estado dicotómicamente aparte de la sociedad y de las clases subalternas, frente a lo cual aparece la trampa de suponer que estas "construyen su historia al margen del Estado, y que este existe al margen y por encima de ellas» (García Linera, 2015a, p. 155). En cambio, García Linera rechaza la premisa de que la acción política transformadora debe marginarse del ámbito de lo estatal, por concebirlo como una expresión aislada de las clases subalternas, irremediablemente determinado por el capitalismo en toda circunstancia.

La alternativa propuesta por el autor radica en la idea de que el Estado debe ser entendido, en primer lugar, como relación social. Esta particular comprensión, ahora bien, debe ser situada en diálogo directo con la obra del intelectual marxista Nicos Poulantzas. En particular, son dos los aportes que el intelectual boliviano está interesado en rescatar del teórico griego: en primer lugar, su comprensión del Estado en tanto condensación de relaciones de fuerza; en segundo, su reflexión por la vía democrática al socialismo —este punto se retomará más adelante-.

La premisa más general que rescata García Linera de la obra tardía de Poulantzas ${ }^{4}$ es aquella de una "condensación material de relaciones de fuerzas entre clases y fracciones de clases», idea ya contenida en distintos textos de la obra de Marx (Poulantzas, 2005, pp. 154). En divergencia de una lectura instrumentalista del Estado y de la idea de una homogeneidad total en su existencia histórica, para García Linera el Estado capitalista moderno condensa también la «subalternidad de las clases» y la conjuga «en estado institucional y simbólico» (García Linera, 2015a, p. 155). Esta condensación no implica, sin embargo, una cooptación simple que se traduce siempre en dominación. Antes bien, para el autor «el Estado es también la comunidad social, los logros comunes, los bienes colectivos conquistados» (García Linera, 2015a, p. 155).

Que el Estado sea una relación social que expresa a la comunidad, como la arriba expuesta, implica, cuando menos, tres puntos innovadores

\footnotetext{
${ }^{4}$ En especial, García Linera dialoga con lo postulado en Estado, poder y socialismo, una de las últimas obras escritas por el pensador griego (Poulantzas, 2005).
} 
en la propuesta del autor boliviano: primero, Linera se refiere a la existencia en el Estado de un contradictorio proceso de monopolización de los bienes comunes; segundo, un constante proceso de estabilización de las relaciones existentes de dominación; y tercero, un permanente proceso de fetichización de la «abstracción real» del Estado.

Frente al primer punto, el Estado tiene una cara «material» representada en instituciones, trámites, burocracia, cárceles, tribunales, poder militar, entre otros (García Linera, 2015a, p. 146); sin embargo, el Estado es «más idea y símbolo que materia» (p. 46), por cuanto las manifestaciones materiales del Estado son solo posibles acorde a un proyecto simbólico más o menos definido que estructura el sentido de tales manifestaciones y les da significado de manera permanente. Como relación dialéctica entre materia e idea, el Estado resulta ser una manera de conocer y darle significado al mundo existente:

De desenvolverse en este tal como ha sido instituido; de saber traducir en acción posible los símbolos del orden dominante instituido y saber desenvolver las acciones individuales o colectivas, ya sea como obreros, campesinos, estudiantes o empresarios, según esas cartas de navegación social que están inscritas en las oficinas, las escuelas, las universidades, el Parlamento, los tribunales, los bancos, etc. (García Linera, 2015a, p. 147).

Para el autor, todo esto implica un continuo proceso de monopolización de los bienes comunes y, más específicamente, de producción del sentido universal de lo común. En síntesis, un contradictorio movimiento entre sintetizar lo común y monopolizarlo. No obstante, este proceso de monopolización está sujeto a una doble tensión histórica. Por un lado, el hecho de que, aunque este proceso de monopolización se muestra permanentemente en beneficio del todo social, lo común en el Estado capitalista «solamente puede constituirse en tanto se instituye para ser simultáneamente usurpado y monopolizado por unos pocos» (García Linera, 2015a, p. 149). Pero, por otro lado, el Estado es también una «trama fluida de relaciones, luchas, conquistas, asedios, seducciones, símbolos, discursos que disputan bienes, recursos y su gestión monopólica» (p. 149). En suma, el Estado —su existencia histórica misma- no está solamente en función de los intereses de las élites que quieren usurparlo, sino que también es producto de largas disputas y ganancias parciales de las distintas fracciones del todo social. 
La clave para el autor radica en que esta trama relacional da cuenta de las relaciones mismas de dominación, resistencia y transformación de la sociedad, inmersas en el sistema capitalista: «el Estado es el constante proceso de estabilización de las relaciones existentes (relaciones de dominación) en los cuerpos y marcos de percepción y de organización práctica del mundo» (García Linera, 2015a, p. 147). ${ }^{5}$ Como ya se advirtió, una de las grandes paradojas de este proceso radica en el hecho de que el monopolio de los recursos comunes $-\mathrm{y}$ el proceso de monopolización en sí mismoes mostrado como común a pesar de que solo beneficia a unos cuantos. Esta paradoja simbólica, ahora bien, lejos de ser arbitraria o aleatoria -o definirse como una «batalla autónoma» confinada al «campo de lo estatal»—, se corresponde más bien con las contradicciones en la producción, la vida social y la cultura.

Allí, en tercer lugar, García Linera identifica una cierta fetichización del Estado y de la dominación de este, tanto de parte de quienes quieren conservar una estatalidad en función de la dominación, como de parte de quienes han querido romper con ella. Incluso, «el Estado-instrumento de las izquierdas del siglo xx es un efecto de esta fetichización de la relación social concebida como cosa con vida propia» (García Linera, 2015a, p. 150).

Dos ambigüedades se asoman hasta acá en el autor: la primera, el hecho de criticar esta fetichización y, a la vez, señalar la necesidad histórica y política de optar políticamente por el Estado es una de las más retadoras -importantes - contradicciones al interior del pensamiento del autor. Para García Linera, optar políticamente por el Estado como una de las vías de la emancipación social reside en el hecho de que sería abstracto pensar que es posible sustraerse completamente - y sustraer la lucha- del ámbito de lo estatal, por la trama relacional que lo vincula de manera ineludible con el todo social.

La segunda ambigüedad, y en consonancia con lo anterior, un punto ciego no suficientemente dilucidado en el planteamiento de García Linera tiene que ver con que toda reflexión por lo común queda circunscrita en el monopolio estatal de lo común. Dicho de otro modo, hay una confusión no suficientemente desarrollada entre lo común en el Estado y lo común más allá del Estado, desconociendo la existencia de otras experiencias en este último sentido. Un ejemplo arriba citado corresponde a lo señalado

\footnotetext{
${ }^{5}$ Énfasis agregado.
} 
por Gutiérrez (2017) a propósito de la vasta diversidad de las experiencias comunitarias-populares bolivianas.

Pese a estas ambigüedades, una de las salidas a las cuales apela el autor es la insistencia en que el Estado, en tanto condensación de relaciones de clase, nunca es completa y homogéneamente favorable a una sola fuerza; por el contrario, el Estado siempre es producto de luchas sociales que generan ganancias parciales de uno y otro bando, por lo cual siempre es «incompleto históricamente» (García Linera, 2015b, p. 14). De hecho, en este principio de incompletitud histórica aparecen intersticios sobre los cuales romper con las fuerzas dominantes que tradicionalmente han detentado el poder estatal, para efectos de propiciar la transformación desde el Estado. ¿De qué forma, entonces, aparecen estos intersticios, acorde a este principio de incompletitud histórica? ¿Cómo se actúa desde el Estado para aprovecharlos en función del cambio histórico? Las siguientes dos secciones reconstruyen brevemente las respuestas del autor a estos interrogantes, resaltando el rol de la democracia y, más aún, la comprensión del autor sobre este concepto.

\section{Democracia}

También pese a las ambigüedades ya señaladas, García Linera ha planteado una preocupación manifiesta hacia la tarea de construir un pensamiento sobre la democracia más allá del Estado (García Linera, 2013). Como hoja de ruta, lo primero que hay que mencionar es que, en contraste con los textos dedicados a «la cuestión del Estado» en sus distintos interrogantes, la producción de García Linera específicamente sobre la democracia es relativamente menor y, de hecho, está casi siempre en función de - o acompañada de- lo que implica construir Estado en la democracia.

Al igual que en su abordaje sobre la cuestión de la nación (Cerrato, 2015), la democracia en García Linera está pensada desde las estructuras históricas y las relaciones de fuerza que componen en el presente los significados teóricos y prácticos de esta. Para el autor, de hecho, «no existe la "verdadera" y definitiva definición de democracia, transhistórica y objetiva» (García Linera, 2013, p. 10). En Democracia, Estado, nación, el autor manifiesta una idea de democracia como concepto en disputa, como convención histórica o «producto provisional de inter-subjetivaciones resultantes de distintos modos de acción comunicativa", en donde, en todo caso, se juegan y se conjugan de manera desigual relaciones de poder y de 
dominación. En tal sentido, pueden encontrarse definiciones dominantes y «legítimas» de democracia, funcionales a un determinado orden de cosas, así como definiciones «subalternas» de democracia, relegadas a la ilegitimidad e invalidadas por el poder dominante. Por tanto, la validez de toda definición de democracia - su «rango de verdad»- es una "contingencia histórica» resultante del estado de la correlación de fuerzas entre las clases en disputa (p. 13). Para el autor estas relaciones de fuerza no surgen de conflictos puramente argumentativos, sino de las contradicciones económicas, culturales y políticas de la sociedad.

En términos de las versiones dominantes de democracia, García Linera señala dos consideraciones críticas: en primer lugar, la democracia es entendida como «herramienta de resultados» (García Linera, 2013, p. 14), esto es, como un conjunto de reglas que posibilita la reproducción ideológica de la legitimidad representativa de las élites en ejercicio del poder a través de su elección constante desde el sufragio de la voluntad general. De esta forma, el hecho democrático queda reducido «a una técnica de agregación de intereses cuya meta es maximizar una utilidad (la selección de elites o resolución de conflictos), similar al papel que en la teoría liberal juega el mercado en la optimización de la asignación de recursos» (p. 20).

De acuerdo con esto, en segundo lugar, esta versión dominante de la democracia se sitúa en un rotundo desencuentro práctico con la complejidad del orden social, especialmente en cuanto a todo lo disidente y a todo lo que escapa de los márgenes de la legitimidad establecida. En el caso boliviano, el conjunto de normas e instituciones previas al proceso revolucionario resultaban obsoletas, desfasadas y opresivas con respecto a la heterogénea y conflictiva composición de luchas, formas culturales disidentes y expresiones populares del panorama político de este país. Para el autor, las instituciones democráticas dominantes parten de la premisa de una abstracta libertad de concurrencia de individuos exactamente iguales, "con facultades para intercambiar bienes políticos al margen de las coerciones extra-políticas» (García Linera, 2013, p. 26).

Frente a lo anterior, García Linera llama a la necesidad de pensar y poner en práctica «otras democracias posibles» que permitan ir más allá de las carencias y limitaciones históricas de una lectura «procedimental y minimalista» de la democracia. Para ello, el autor provee una definición general basada en la idea de una forma política de organizar y gestionar el 
«bien común» de una sociedad en términos de comunidad. Más aún, una democracia en la que el Estado es solo un momento - y no el fin últimode la política. Esto incluye la riqueza comunitaria e intercultural dada en las experiencias asamblearias de los movimientos sociales e, incluso, en la perspectiva del buen vivir:

Se puede entender la democracia como una manera de organizar la gestión del bien común de una sociedad, el modo de esa gestión, la amplitud de ese bien común y las propias características de la comunidad que quiere definirse en torno a ese bien. En este sentido, es una forma política de proceder sobre los recursos e intereses colectivos; es un proceso de renovación de los modos de decidir sobre ese bien común, es una voluntad para redistribuir los recursos comunes; es una deliberación sobre cuáles son esos recursos y necesidades que deben ser objeto de atención y, ante todo, una continua producción del «común» que desea, acepta y pugna por existir como comunidad (García Linera, 2013, p. 28).

Pese a que se plantea la necesidad de pensar «otras democracias posibles» basadas en un ejercicio de subjetivación política abierto a la contingencia, al conflicto y a la revocabilidad de lo estatal, la confusión entre lo común y el monopolio estatal de lo común se reactiva en las líneas dedicadas por el autor a la cuestión de la democracia, en especial, por cuenta del hecho de que el carácter contingente y conflictivo de lo democrático está en función de configurar, replantear, moldear y mejorar lo públicoestatal —o el monopolio estatal de lo común-, pero sin una reflexión más detenida sobre las posibilidades de apertura contingente de lo común en otros registros. García Linera parece estar sujeto a la ambigüedad de querer tomar nota de una idea de democracia como actividad de subjetivación contingente, nunca clausurada y conflictiva, pero de encerrar esta premisa de contingencia dentro de los márgenes de lo público estatal.

Aun así, queda por recorrer un elemento significativo para entender la manera en que el planteamiento de García Linera pretende ser una alternativa que responde a la contradicción entre la pregunta por cómo actuar desde el Estado y la necesidad de acoger la demanda política y teórica de la revocabilidad y transitoriedad de lo estatal, sujeta a la permanente actividad política de una sociedad democrática en constante litigio. En este punto, la elegida por el autor para abordar esta tensión aparece de nuevo con Poulantzas (2005). 


\section{Estado y democracia, o la vía democrática al socialismo}

Dijimos al inicio de este texto que el segundo elemento que retoma García Linera de su interpretación de Poulantzas es aquel de la vía democrática al socialismo. En la apuesta del autor griego se conjugan una lectura heterodoxa del marxismo althusseriano, una actualización del debate entre Lenin y Rosa Luxemburgo a propósito de la relación de los sóviets con el Estado revolucionario y un intenso debate con la filosofía política francesa posestructuralista (Reyna, 2017). Según Michael Löwy (2014, diciembre 18), Poulantzas invoca en especial a Luxemburgo para pensar la relación entre democracia y Estado más allá de un modelo binario en el que sea solo uno de los dos el elemento «decisivo» de la revolución: «El punto que Rosa hizo - y que Nicos recogió, y luego fortaleció- se refiere a la necesidad de combinar tanto la democracia representativa como la directa en el proceso revolucionario».

La dicotomía entre estatismo jacobino y basismo autogestionario como dos opuestos inconciliables es abordada por Poulantzas yendo directamente al debate entre Lenin y Luxemburgo a propósito de la relación entre consejismo y Estado (Poulantzas, 2005). Ni Estado como pedestal aislado

[212] de las masas ni esencialismo de las masas como único camino posible para la revolución. La tesis de Poulantzas sobre la vía democrática al socialismo requiere entender los desgarres y determinaciones mutuas entre Estado y «masas», no como dos homogéneos y monolíticos campos separados, sino en cuanto a la forma en que se insertan y existen en un determinado estado de las relaciones de clase.

García Linera reinterpreta este planteamiento dialéctico sobre democracia y Estado, de donde se desprende que no hay «socialismo» sino en el seno mismo de esta idea de permanencia de la contradicción y de una temporalidad constante e inacabada. El Estado, como objetivación de la actividad democrática, debe servir para potenciar la democracia y eso implica una apertura irreductible a la posibilidad de que la democracia destruya, transforme y construya siempre al Estado, de nuevo, este siendo solo un momento de aquella. No se trata de una eliminación gradual del Estado dispuesta, teleológicamente, a su destrucción última, en cambio, se trata de un estatuto de revocabilidad y reconfiguración permanente a manos de la actividad constante del demos. 
De cómo se especifica esta particular comprensión de la tensión perseverante entre Estado y democracia en el pensamiento del autor, se pueden destacar tres elementos: primero, como ya se mencionó, la existencia permanente de intersticios en el Estado, en tanto entramado de relaciones sociales, intersticios sobre los que fluye la subversión democrática. Estos intersticios son más reducidos y oponen más resistencia en el marco de un Estado anquilosado en la defensa propia, pero se amplían y se vuelven constitutivos en la estructura misma del «Estado nuevo», al punto de ubicar la revocabilidad más lejos de lo excepcional y más cerca de la cotidianidad democrática misma. Se asoma, entonces, una tensión permanente entre la consecución de una estabilidad relativa - no es ideal, en todo caso, un estado en permanente crisis- y la necesidad de potenciar una democracia siempre abierta a la revocatoria de lo instituido.

Segundo, el hecho de que la búsqueda de este equilibrio sea mucho más que una formalidad y no se dé en condiciones históricas, económicas, políticas, culturales y sociales «ideales», sino que se halle inserta en la variante composición de las relaciones de fuerza y de clase, en donde los conflictos van más allá de ser cuestiones meramente comunicativas para involucrar formas de dominación, opresión y explotación en el ser social. Conseguir este equilibrio, entonces, es más difícil por cuenta de que encontrar una justa medida entre Estado y democracia es un ejercicio sometido a la amenaza de los intereses de quienes dominan por perpetuar sus privilegios y de quienes son dominados por subvertir el estado presente de su opresión. Esta «justa medida», lejos de ser un punto de plena estabilidad, parte de reconocer el carácter irresoluble de las contradicciones y de avanzar, hacia la emancipación, sobre la base de nuevos desafíos políticos y sociales que aparecen de manera permanente.

Tercero, las amenazas a la búsqueda de un equilibrio entre un Estado nuevo y una democracia activamente subversiva no solo se ubican en las relaciones de fuerza «locales» de determinado contexto nacional —el Estado boliviano, la democracia boliviana y la nación boliviana-, sino que están sujetas a la estructura de producción global y la geopolítica global del capitalismo contemporáneo, por lo que la lucha por una vía democrática al socialismo, lejos de encasillarse en el localismo o nacionalismo, debe plantearse en perspectiva de disputa global (García Linera, 2015b, p. 14). La fragmentación de las luchas, por el contrario, termina siendo absorbida 
de algún modo por la organización de la producción y de la geopolítica internacional: «A la larga el capitalismo vuelve a reproducirse absorbiendo y alimentándose de esa energía fragmentada. El activismo aislado se convierte sin que uno lo quiera o lo desee en una fuerza productiva del capitalismo porque lo ayuda a regenerarse, lo ayuda a potenciarse; sin desearlo lo ayuda a expandirse» (García Linera, 2015b, p. 16).

Es la idea de socialismo como un «campo de batalla dentro de cada territorio nacional entre una civilización dominante, el capitalismo [y] la nueva civilización comunitaria emergente» (p. 69), el trasfondo sobre el que es pensada la inclausurable relación entre Estado y democracia. Para García Linera (2013), el socialismo no es la llegada de una sociedad plenamente reconciliada consigo misma, en donde al conflicto y a la contradicción se les suprime. Es, en cambio, el espacio para disputar la búsqueda por una democracia no asfixiada por el Estado ni por las determinaciones históricas locales ni por las globales que hacen que el Estado resulte ser asfixiante. Por eso el socialismo es «desborde democrático, es socialización de decisiones en manos de la sociedad auto-organizada en movimientos sociales» (p. 69). $\mathrm{Y}$ recrear tal proyecto civilizatorio alternativo, tal "comunidad universal», debe situar la irreductible contradicción democracia-Estado en todas sus determinaciones materiales, simbólicas y a escala global. Por eso, «al final, la comunidad real será universal, planetaria o no será nada» (p. 69).

\section{Ambigüedades}

Retomando las menciones críticas hechas a lo largo del artículo, hay cuatro ambigüedades que resaltan en la argumentación de García Linera, todas ellas a propósito de la alternativa teórica puesta por el autor en términos de la relación entre Estado y democracia.

Primero, García Linera parece asumir con ligereza una conceptualización más detallada sobre lo que implica lo común más allá del Estado, lo que deja abierta la pregunta de si afirmar que el Estado es un espacio de monopolización de lo común implica necesariamente que la lucha por lo común -y en general, lo común en sí mismo- quede exclusivamente relegado al ámbito de lo Estatal. García Linera parece estar confundiendo permanentemente $-y$ habría que preguntarse si esto puede ser entendido en el marco de la necesidad de legitimar la lucha desde el Estado- estas dos dimensiones. Esto resta posibilidades para pensar más 
complejamente las formas de recrear lo común más allá del Estado y de entender que si bien lo común también se disputa desde la estatalidad, esta disputa no se circunscribe exclusivamente a aquel. Esta ambigüedad también emerge en su entendimiento sobre el carácter litigante de la democracia y la revocabilidad permanente a la cual está sujeto el ámbito de lo estatal en ella. Pese a que pretende perfilar al Estado solo como un momento de lo democrático, el autor parece priorizar permanentemente al Estado como el momento crucial en la construcción de lo común.

Segundo, estrechamente conectado a lo anterior, es la forma en que García Linera ha conceptualizado lo común y el problema de la nación sobre la tensión entre las perspectivas de lo nacional-popular y lo comunitario-popular, para lo cual varios críticos han señalado, fundamentalmente, el riesgo de ponderar el primer horizonte - nacionalpopular- sobre el segundo. Silvia Rivera Cusicanqui (2015), por ejemplo, entiende la propuesta de García Linera como una «concepción autoritaria e idealista de la Nación» (p. 9) sobre la cual hay un uso pragmático e instrumental de «lo étnico» que no reconoce la diversidad boliviana sino de una manera formalista y funcional al oficialismo. Para la autora, el gobierno del MAS reproduce un discurso de «etnicidad estratégica» que parte de «un pensamiento dualista que postula esencias antes que procesos o prácticas colectivas» (p. 10), lo que deriva en la pretensión de consolidar una «identidad primordial» de «adhesión fuerte» (p. 10). Peter Baker (2015a), por su parte, señala que García Linera permanece atrapado en una lógica que por momentos parece reproducir un modelo clásico de soberanía nacional y, por tanto, una propuesta de plurinacionalidad que va en detrimento de las identidades singulares en Bolivia.

En esta última línea, otra de las autoras críticas con la argumentación de García Linera es Madelena Cerrato (2015), quien señala que el uso singular que García Linera le da a la idea de nación está sujeto a una doble contradicción. Por un lado, el hecho de que el autor no pueda renunciar a la nación en virtud del potencial ideológicamente subversivo de «la subjetivación colectiva nacional» en el marco de una geopolítica antiimperialista: «La forma-nación evoca la lucha anti-imperialista, la lucha en contra de la dominación y la opresión por independencia e igualdad y, como tal, juega [sic] un rol estratégico en la oposición al trans-nacionalismo del capitalismo global contemporáneo» (p. 336). Pero, por otro lado, Cerrato 
señala que, al esbozar un insistente objetivo de unidad nacional, García Linera «no está listo para renunciar a la construcción de un sujeto histórico de la revolución colectivo y soberano, la construcción de un sujeto contrahegemónico capaz de operar activamente el cambio y a la vez resignificar la soberanía del Estado boliviano, manteniéndolo vivo [y con ello] reclamar la legitimidad de la forma Estado nación» (p. 336).

Valorar el uso estratégico de la Nación en términos del antiimperialismo y la recreación de una "gobernabilidad» revolucionaria tiene dos caras. Cerrato —al igual que Baker (2015b) - está interesada en una crítica poscolonial de esta contradicción, lo que dejaría atrapado a García Linera en una insalvable «lógica de la soberanía» y en un «substancialismo teleológico del sujeto de la revolución». En tal sentido, los alcances críticamente transformadores serían menores que las implicaciones en términos de la reproducción de sistemas de creencias dominantes y aún insertados en la colonialidad del saber. En últimas, los autores señalan la carga teórica eminentemente moderna y colonial de una concepción autoritaria e idealista de la nación (Moreiras, 2015, p. 280).

La ambigüedad, en síntesis, se traduce en que pese a la legitimidad de

[216] la preocupación de García Linera de responder a la dispersión de las luchas bolivianas a través de un proyecto de construcción de hegemonía y de articulación política, los críticos del oficialismo han señalado que el hecho de que esta preocupación se haya traducido en una propuesta simbólica, principalmente centrada en lo nacional-popular denota aún la reproducción de paradigmas coloniales y de políticas identitarias que pueden resultar violentas con la singularidad de las comunidades que habitan el territorio boliviano.

Lo anterior, al mismo tiempo, es bastante útil para entender la tercera ambigüedad que se quiere señalar aquí. La forma en que en el Estado se consolidan estos imaginarios colectivos y esta apuesta por lo común se concreta también, como el mismo García Linera lo acepta, en dispositivos, instituciones, canales de transmisión cultural e ideológica, aparatos burocráticos, entre otros. Si bien el Estado no es reductible o simplificable a estos últimos -y, de nuevo, ahí aparece el gran aporte del autor en el contexto latinoamericano al analizar la dimensión relacional, de clase y la dimensión simbólica de la estatalidad-, la gran ausencia en el planteamiento de García Linera es la de cómo teorizar más elaborada y detenidamente la relación que 
esta dimensión simbólica y relacional tiene con los aparatos de Estado y la forma en que se concreta en acciones y relaciones de poder respecto a otros actores colectivos. En suma, no hay un análisis más detallado del Estado como actor colectivo y como aparato, y de la forma en que esto se traduce en una serie de relaciones - potencialmente violentas - con otros actores y movimientos.

Finalmente, hay una ambigüedad referida a la gran ausencia de una economía política del Estado boliviano contemporáneo, en especial, en términos de sus rentas extractivas y su relación con la economía política global. Poulantzas (2005) fundamenta su comprensión del Estado en un extenso trabajo sobre la relación entre estructura y superestructura y la forma en que distintas tradiciones del marxismo han conceptualizado mecánicamente esta relación, entendiendo al ámbito de lo económico como independiente y autónomo con respecto al ámbito de lo político. Poulantzas está en contra de la idea de que estos dos sean dos autónomos irreductibles, para cada uno de los cuales haya disponible tanto una «teoría general de la economía» como una «teoría general del Estado». Como ya se dijo, la vía escogida por Poulantzas para problematizar y desvirtuar esta dicotomía parte, en primer lugar, de un extendido trabajo sobre lo que significan la economía, el Estado y, por supuesto, la relación entre ambos.

La forma en que la argumentación ofrecida por García Linera dialoga con lo anterior evade una reconstrucción más detenida del ámbito de lo económico —en particular, de las manifestaciones contemporáneas del capitalismo en Bolivia- y se concentra de forma más minuciosa en una teorización del Estado como lugar de la transformación y la revolución, valga decir, al menos en tres aspectos: i) lo que significa este en términos tácticos y estratégicos; ii) la dimensión simbólica del Estado, más allá de los aparatos estatales más concretos e inmediatos; iii) la relación del Estado con los movimientos sociales y las luchas «de base».

Sin embargo, algunas tesis como la de un «Estado compensatorio» o «revolución pasiva», para el caso de la experiencia del gobierno del MAS (Modonesi, 2012; Webber, 2015), permiten ver que la estructura económicopolítica concreta de manera problemática varios de los planteamientos allí inmersos y provee un marco de circunstancias que no pueden omitirse para tales efectos: aunque en este periodo hubo una redistribución relativa de la renta que contribuyó a una disminución igualmente relativa de los índices 
de pobreza extrema, este proyecto nunca cuestionó de manera significativa las relaciones de clase en los ámbitos local y global sobre las que estaban fundadas las rentas extractivas y los buenos réditos allí obtenidos por cuenta de un boom de las commodities, orientado, en especial, por el dinamismo productivo de China en la primera década del siglo xxı (Webber, 2015). Incluso en términos teóricos, este análisis económico-político resulta igualmente fundamental para concretar y vislumbrar potencialidades y límites a las contradicciones sobre las que se mueven los flujos de condensación de fuerzas y de relaciones de clase, tanto en el Estado como en la democracia $-\mathrm{y}$ eventualmente, en la idea de un proyecto de socialismo democrático- ${ }^{6}{ }^{6}$

\section{Conclusión}

En suma, el pensamiento de García Linera busca responder, en la sociedad boliviana contemporánea, al problema de cómo pensar una estatalidad transformadora y en permanente relación tensional con movimientos sociales y de base, con la lógica del Estado-nación en el presente histórico boliviano, con la perspectiva popular de la democracia y con las restricciones del capitalismo global en Bolivia. Para ello, el autor construye uno de los planteamientos más significativos y visibles en la teoría

[218] política latinoamericana contemporánea sobre la complejidad de pensar una acción política desde el Estado, en la perspectiva de su relación con las otras instancias de la vida social. Como se expuso, un punto privilegiado para recorrer tal planteamiento es el de la concepción que tiene el autor a propósito de la relación entre Estado y democracia.

Sin embargo, García Linera está sujeto a una serie de dificultades y ambigüedades para pensar la relación entre acción política transformadora desde lo estatal y acción política transformadora más allá de lo estatal. Por un lado, el autor ubica una serie de retos teóricos y políticos pensados en el presente en términos de la relación entre Estado y democracia que refieren una apertura relativa a la contingencia y a una temporalidad de no clausura que configura y reconfigura permanentemente lo estatal, agenciado por un permanente proceso de subjetivación democrática. Pero, por otro lado,

\footnotetext{
${ }^{6}$ El tema de la economía política del extractivismo en Bolivia es bastante amplio y trabajado y supera los alcances de este texto, si bien estamos interesados en sugerir la forma en que ocupa una ambigüedad en el planteamiento del autor.
} 
hay una clara subteorización en la propuesta del autor, expresada en una constante confusión entre lo común y lo público, una identificación de lo común con lo público-estatal y una idea de lo democrático, principalmente como potencia litigante y revocadora, que tendría que ocuparse, de manera más detenida, de cómo esto opera a la luz de las contradicciones históricas que le competen a la sociedad boliviana en el presente.

\section{Referencias bibliográficas}

1. Baker, Peter. (2015a). The Phantom, The Plebeian and the State: Grupo Comuna and the Intellectual Career of Álvaro García Linera. Viewpoint Magazine. Dossier: Álvaro García Linera: A Bolivian Marxist Seduced. Retrieved from https:// www.viewpointmag.com/2015/02/25/the-phantom-the-plebeian-and-the-state-grupocomuna-and-the-intellectual-career-of-alvaro-garcia-linera/

2. Baker, Peter. (2015b). Can the State Learn to Live Well? Álvaro García Linera as an Intellectual of the State and Interpreter of History. Culture, Theory and Critique, 56 (3), pp. 283-296. DOI: 10.1080/14735784.2015.1012684

3. Cavooris, Robert. (2015). Introduction: A Bolivian Marxist Seduced. Viewpoint Magazine. Dossier: Álvaro García Linera: A Bolivian Marxist Seduced. Retrieved from https://www.viewpointmag.com/2015/02/25/introduction-a-bolivianmarxist-seduced/

4. Cerrato, Madelena. (2015) Nation Form, Community Form: Nationalisation and Dialectic in García Linera's Thought. Culture, Theory and Critique, 56 (3), pp. 333-348. DOI: 10.1080/14735784.2015.1066262

5. Crabtree, John \& Whitehead, Laurence. (2008). Unresolved Questions: Bolivia, Past and Present. Pittsburg: University of Pittsburg. DOI: 10.2307/j.ctt5vkfjq

6. Cunha Filh, Clayton. (2014). El «proceso de cambio» en Bolivia: un balance de ocho años. Tinkazos, 17 (35), pp. 137-153.

7. Farthing, Linda \& Kohl, Benjamin. (2014). Evo's Bolivia: Continuity and change. Austin: University of Texas.

8. García Linera, Álvaro. (2008). La potencia plebeya. Acción colectiva e identidades indígenas, obreras y populares en Bolivia. Buenos Aires: Clacso.

9. García Linera, Álvaro. (2011). Las tensiones creativas de la revolución. La quinta fase del Proceso de Cambio. La Paz: Vicepresidencia del Estado Plurinacional.

10. García Linera, Álvaro. (2013). Democracia, Estado, nación. La Paz: Vicepresidencia del Estado Plurinacional.

11. García Linera, Álvaro. (2015a). El Estado y la vía democrática al socialismo. Nueva Sociedad, 259. Recuperado de https://nuso.org/articulo/el-estado-y-lademocratica-al-socialismo/ 
12. García Linera, Álvaro. (2015b). Socialismo comunitario: un horizonte de época. La Paz: Vicepresidencia del Estado, Presidencia de la Asamblea Legislativa Plurinacional.

13. García Linera, Álvaro; Prada, Raúl; Tapia, Luis y Vega Camacho, Oscar. (2010). El Estado. Campo de lucha. La Paz: Clacso.

14. Gutiérrez, Raquel. (2017). Horizontes comunitario-populares. Producción de lo común más allá de las políticas estado-céntricas. Madrid: Traficantes de Sueños.

15. Holloway, John. (2002). Cambiar el mundo sin tomar el poder. El significado de la revolución hoy. Buenos Aires: Herramienta.

16. Löwy, Michael. (2014, diciembre 18). Nicos Poulantzas tel que je l'ai connu. Contretemps. Revue de critique communiste. Recuperado de https://www. contretemps.eu/nicos-poulantzas-tel-que-je-lai-connu-par-michael-lowy/

17. Modonesi, Massimo. (2012). Revoluciones pasivas en América Latina: Una aproximación gramsciana a la caracterización de los gobiernos progresistas de inicio del siglo. En: Thwaites Rey, Mabel (ed.). El Estado en América Latina: continuidades y rupturas (pp. 139-167). Buenos Aires: Clacso.

18. Moreiras, Alberto. (2015). Democracy in Latin America: Álvaro García Linera, an Introduction. Culture, Theory and Critique, 56 (3), pp. 266-282, DOI: 10.1080/14735784.2015.1070976

19. Poulantzas, Nicos. (2005). Estado, poder y socialismo. México, D. F.: Siglo xxı. 20. Prada, Raúl. (2009). Subversiones indígenas. La Paz: Muela del Diablo.

[220] 21. Prada, Raúl. (2013, enero 16). Defensa crítica del proceso. Rebelión. Recuperado de http://www.rebelion.org/noticia.php?id = 162342

22. Reyna, Jaime. (2017). El rechazo de lo inerte: Álvaro García Linera y sus primeras lecturas de Marx. Revista Kavilando, 9 (2), pp. 457-470.

23. Rivera Cusicanqui, Silvia. (2012, enero 31). Del MNR a Evo Morales: disyunciones del estado colonial. Bolpress. Recuperado de http://www.bolpress.com/ print.php?Cod $=2012123104 \& p=1$

24. Rivera Cusicanqui, Silvia. (2015). Mito y desarrollo en Bolivia: el giro colonial del gobierno del MAS. La Paz: Piedra Rota.

25. Tapia, Luis. (2010). El Estado en condiciones de abigarramiento. En: García Linera, Álvaro; Prada, Raúl; Tapia, Luis y Vega Camacho, Oscar. El Estado. Campo de lucha (pp. 97-128). La Paz: Comuna, Muela del Diablo.

26. Webber, Jeffery. (2011). From Rebellion to Reform: Class Struggle, Indigenous Liberation and the Politics of Evo Morales. Chicago: Haymarket.

27. Webber, Jeffery. (2015). Burdens of a State Manager. Viewpoint Magazine. Dossier: Álvaro García Linera: A Bolivian Marxist Seduced. Retrieved from https:// www.viewpointmag.com/2015/02/25/burdens-of-a-state-manager/ 